\title{
SAR-SHARPENING IN THE KENNAUGH FRAMEWORK APPLIED TO THE FUSION OF MULTI-MODAL SAR AND OPTICAL IMAGES
}

\author{
A. Schmitt ${ }^{1}$ and A. Wendleder ${ }^{2}$ \\ ${ }^{1}$ Munich University of Applied Sciences, Department of Geoinformatics, Karlstraße 6, D-80333 Munich, schmitt@hm.edu \\ ${ }^{2}$ German Aerospace Center (DLR), Earth Observation Center, Oberpfaffenhofen, D-82234 Weßling, anna.wendleder@dlr.de
}

\section{Commission I, WG I/6}

KEY WORDS: SAR, Optical, Image fusion, Image sharpening, Polarimetry, Multispectral imaging, Multi-sensor data fusion

\begin{abstract}
:
The Kennaugh framework turned out to be a powerful tool for the preparation of multi-sensor SAR data during the last years. Using intensity-based (an-) isotropic diffusion algorithms like the Multi-scale Multi-looking or the Schmittlets, even robust preclassification change detection from multi-polarized images is enabled. The only missing point so far, namely the integration of multi-mode SAR data in one image, is accomplished in this article. Furthermore, the Kennaugh decomposition is extended to multispectral data as well. Hence, arbitrary Kennaugh elements, be it from SAR or optical images, can be fused. The mathematical description of the most general image fusion is derived and applied to four scenarios. The validation section considers the distribution of mean and gradient in the original and the fused images by the help of scatter plots. The results prove that the fused images adopt the spatial gradient of the input image with a higher geometric resolution and preserve the local mean of the input image with a higher polarimetric and thus also radiometric resolution. Regarding the distribution of the entropy and alpha angle, the fused images are always characterized by a higher variance in the entropy-alpha-plane and therewith, a higher resolution in the polarimetric domain. The proposed algorithm guarantees optimal information integration while ensuring the separation of intensity and polarimetric/spectral information. The Kennaugh framework is ready now to be used for the sharpening of multi-sensor image data in the spatial, radiometric, polarimetric, and even spectral domain.
\end{abstract}

\section{INTRODUCTION}

Earth observation satellites with their diversity of sensors provide a variety of spectral, geometric, temporal, and radiometric resolutions. Their rising number raises the issue of image fusion in order to enhance interpretation capabilities of image features (Pohl and van Genderen, 1998; Abdikan et al., 2008) and to reduce the amount of data at the same time. For instance, Pan-Sharpening combines a high resolution panchromatic image with a low resolution multispectral image and creates a multispectral image with higher-resolution features. This improves the thematic interpretation enormously and can be seen as state of the art nowadays. Cliche et al. (1985) demonstrated that the spatial resolution of $20-\mathrm{m}$ multispectral SPOT data can be increased by integrating the $10-\mathrm{m}$ panchromatic channel. Chavez et al. (1991) compared three different methods of Pan-Sharpening and found that distortions of the spectral characteristics using a High-Pass Filter were minimal. Equally, image fusion of panchromatic and SAR data enhances the understanding and classification of objects due to the combination of two disparate data: on the one hand, optical data with information on the reflective and emissive characteristics of the earth's surface features and SAR data with information on surface roughness, texture, and dielectric properties on the other hand (Pohl and van Genderen, 1998; Amarsaikhan et al., 2010). Amarsaikhan et al. (2010) used optical and SAR data for the enhancement of urban features and demonstrated that multi-source information could significantly improve the interpretation and classification of land cover types. However, image fusion of only SAR images, possibly acquired in different frequencies or polarizations, is not well established in practice. The so-called SAR-Sharpening primarily denotes an increase of the spatial resolution. Depending on surface roughness, texture, and dielectric properties of an object, each frequency and each polarimetry underlies a completely different scattering behaviour. Additionally, SAR images are influenced by high and diverse noise content: additive (white) noise and the multiplicative speckle effect. Thus, the basic idea of combining SAR images with different frequencies and polarizations is a radiometric stabilization without reduction of the spatial resolution. With respect to the interpretation of backscatter values, this immediately leads to an increase of the information content (Simone et al., 2001; Farina et al., 1996). This image fusion is novel and promising as it supports the understanding and interpretation of SAR image features due to different electromagnetic signatures. Simone et al. (2001) combined multi-frequency, multi-polarized, and multi-resolution intensity images incoherently using the discrete wavelet transform. The classification results underlined an improved discrimination of land cover types. Weissgerber (2016) combined a singlepolarized high-resolution TerraSAR-X image and a quadpolarized coarser resolution TerraSAR-X image acquired under interferometric conditions, thus coherent. The goal was to exploit the scattering mechanisms of polarimetric SAR images even in fine-structured urban environments. The method consequently enhanced the spatial resolution of point-like targets while keeping their polarimetric behaviour.

Our approach proposes a versatile SAR-Sharpening in the Kennaugh framework. The idea is to establish a simple but consistent mathematical description which supports both the fusion of several SAR images and the fusion of SAR with optical data. The Kennaugh framework is already in use for polarimetric decomposition and data preparation and has proven to be suitable in diverse applications (Schmitt and Brisco, 2013; Moser et al., 2015; Bertram et al., 2016). Its advantage is the consistent preparation of all SAR data independent of sensor, mode and polarization. The final product consists always of geocoded, calibrated, and normalized Kennaugh elements, i.e. one intensity measure and up to nine polarimetric measures. The existing framework is expanded to the integration of optical images as well. Hence, SAR and optical Kennaugh elements are defined 


\begin{tabular}{|c|c|c|c|c|c|c|}
\hline Scenario & Acquisition date & Sensor & Mode & Polarization & Looks & Target Grid (m) \\
\hline \multirow[b]{2}{*}{1} & 12.05 .2017 & ALOS-PALSAR-2 & StripMap & $\mathrm{HH} / \mathrm{VV} / \mathrm{HV} / \mathrm{VH}$ & 0.5 & \multirow[b]{2}{*}{$2 \times 2$} \\
\hline & 20.04 .2017 & TerraSAR-X & SpotLight & HH/VV & 1.1 & \\
\hline \multirow{2}{*}{2} & 22.11 .2014 & TerraSAR-X & StripMap & $\mathrm{HH} / \mathrm{VV}$ & 1.5 & \multirow{2}{*}{$2.5 \times 2.5$} \\
\hline & 03.12 .2014 & TerraSAR-X & StripMap & $\mathrm{VV} / \mathrm{VH}$ & 1.5 & \\
\hline \multirow{2}{*}{3} & 10.05 .2017 & Aerial Camera & - & - & 25 & \multirow{2}{*}{$1 \times 1$} \\
\hline & 12.05 .2017 & ALOS-PALSAR-2 & StripMap & $\mathrm{HH} / \mathrm{VV} / \mathrm{HV} / \mathrm{VH}$ & 0.1 & \\
\hline \multirow[b]{2}{*}{4} & 14.10 .2017 & Sentinel-2 & - & - & 1.0 & \multirow{2}{*}{$10 \times 10$} \\
\hline & 15.10 .2017 & Sentinel-1 & Interferometric Wide Swath & $\mathrm{VV} / \mathrm{VH}$ & 2.1 & \\
\hline
\end{tabular}

Table 1. Sensor characteristics and acquisition parameters of the available data sets for the four scenarios.

which can be fused to one image. The fused images benefit from the increased resolution in both the spatial and polarimetric/spectral domain. Four scenarios are designed in order to prove the added value of the fused image: (1) traditional SARSharpening in the spatial domain, (2) SyntheticQuadPol, (3) SAR-Sharpening involving a pan-chromatic image, and (4) the fusion of SAR and optical features provided by the Sentinel-1\&2.

\section{TEST SITES AND REMOTE SENSING DATA}

This section introduces the four application scenarios for the following methodology (Tab. 1). Scenarios 1 and 2 fuse two SAR images whereas scenario 1 enhances the spatial resolution of an ALOS-ALSAR-2 QuadPol StripMap (SM) by the combination with a TerraSAR-X SpotLight (SL). The test site covers the estuary of the Lech into the Danube near Rain am Lech in Bavaria, Germany. This landscape is characterized by canalized river courses, artificial lakes, floodplain forests, agricultural areas, and settlements. Scenario 2 improves the polarimetric resolution by fusing dual-co- and dual-crosspolarized StripMaps (SM) of TerraSAR-X to a so-called "SyntheticQuadPol" image. The test site covers the northern part of Khayelitsha which is a district in Cape Town, South Africa, with formal settlements, planned Townships and informal, completely unorganized settlements in a relatively dry environment. The remaining scenarios concern the fusion of SAR images with optical data. Scenario 3 combines a QuadPol acquisition of ALOS-PALSAR-2 with an Arial Image over Langwasser which is a quite new district in Nuremberg, Germany. This test site contains very diverse urban structure types: residential buildings (with varying orientation), parks, the southern cemetery, the Nuremberg exhibition area, a railroad shunting yard, and industrial buildings. Scenario 4 benefits from the synergy of Sentinel-1 and Sentinel-2 by introducing SAR intensity into an optical image and vice versa. The test site is located near Osterseen in Bavaria, Germany. This area is an extensive wetland with numerous swamp lakes popular as local recreation area.

\section{THE KENNAUGH FRAMEWORK}

Traditional image fusion algorithms deals with one target and one warp image (Brown, 1992). The target image commonly defines the reference for the final fused image in terms of geometry and radiometry and with respect to the polarimetric and/or spectral bands. Our approach defines an independent, earth-fixed and practice-oriented reference frame, in which all input images were transformed as follows.

\subsection{Geometric frame}

In most applications, earth-fixed coordinates are required in order to combine the remotely sensed information with geoinformation data bases; hence, satellite images have to be geocoded in a pre-processing step. Thanks to the high accuracy of today's positioning systems, the satellites orbits can be predicted with an accuracy of about $10 \mathrm{~m}$, measured with $1 \mathrm{~m}$ and adjusted (in a post-processing step) with about $0.1 \mathrm{~m}$ accuracy (Peter et al., 2017). With respect to the common pixel sizes of $10 \mathrm{~m}$ at minimum in the Sentinel-1 mission (in square ground-range pixels with a reasonable number of looks), the orbit deviation delivered with the image ranges around a tenth of the pixel size. Thus, geocoding is simply possible using orbit data and a digital elevation model. Because of the weak influence of atmospheric disturbances on the microwave band, SAR acquisitions can be projected on the earth's surface by solving the Doppler equation for each range line with the accuracy of a few meters or even less (Schubert et al., 2015). Only the geocoding of very high resolution SAR acquisitions or the interferometric analysis of image stacks requires the consideration of atmospheric effects. Optical bands on the contrary are much more affected by refraction. As the influence in the geometry increases with the incidence angle, steep (near nadir) acquisitions are generally preferred. In the case of Sentinel-2, the maximum incidence angle is only $10^{\circ}$. Because of its push broom characteristics, the central projection equation can be solved for each row neglecting further distortions. The gained geolocation accuracy does not exceed a few meters according to recent studies (Vajsova and Åstrand, 2015).

\subsection{Radiometric frame}

As the pixels are geocoded onto the earth's surface, equally the radiometric frame should consider the horizontal area. For SAR acquisitions, this means that $\sigma_{0}$ is calculated using the $\beta_{0^{-}}$ calibrated intensity values and the local incidence angle (Schmitt et al., 2015) being well aware of recent, more sophisticated methods that are preferable for rough terrain (Small, 2011). The common models only concern the backscatter intensity. All polarimetric channels are treated the same way, although the impact of target orientation on polarimetric measurements is well-known (Li et al., 2015). In consequence, the applied calibration does not change the polarimetric properties (see chapter 3.3). Optical data of Sentinel-2 are already delivered as Top-Of-Atmosphere calibrated products (Level 1C). The provided image value thus directly reflects a multiple of the quotient of the measured intensity to the solar illumination. Some images are also available as Bottom-Of-Atmosphere (Level 2A) products. Those are already corrected for atmospheric influences as far as possible (ESA, 2018). It is recommended to use the best calibration variant available, though the influence on the fusion algorithm is almost negligible. The only important characteristic is that all data sets (SAR and optical data) are normalized to reflectance values referring to the horizontal plane similar to $\sigma_{0}$.

\subsection{Polarimetric frame}

SAR sensors always transmit polarized microwaves in order to enable coherent measurements needed for the synthetic aperture 
calculation. Today's sensors typically measure $S_{H H}, S_{H V}, S_{V H}$, or $S_{V V}$, the so-called elements of the Sinclair matrix $S$ in linear polarization with horizontally or vertically oriented transmission and reception (Moreira et al., 2013). The included absolute but random phase impairs the direct interpretation of these complex values. Therefore, different methods of forming intensity measurements by removing the absolute phase have been developed: inter alia the coherency matrix, the covariance matrix, and the Mueller matrix which denotes the linear transform of the real Stokes vector. In the special case of a monostatic SAR system it reduces to the Kennaugh matrix (Schmitt and Brisco, 2013) consisting of the total intensity $\mathrm{K}_{0}$ and up to nine polarimetric Kennaugh elements $K_{i}$. These can be divided by the total intensity and result in the so-called normalized Kennaugh elements $\mathrm{k}_{\mathrm{i}}$ ranging in-between -1 and +1 . The total intensity $\mathrm{K}_{0}$ can be related to the norm intensity of 1 by the TANH scaling and results in the normalized intensity element $\mathrm{k}_{0}$ with the identical data range (Schmitt et al., 2015). The interpretation of polarimetric elements is quite simple. The value zero means "no polarimetric information". Any deviation from zero indicates polarimetric information. The sign shows the direction, for example positive values of $k_{3}$ stand for a higher even-bounce scattering and negative values for a higher odd-bounce scattering in the dual-co-polarized case (Moser et al., 2016). The strength of the effect can be expressed in the unit-less TANH measure or traditionally in decibel. The normalized Kennaugh elements hence enable the separation of intensity from polarimetry (Ullmann et al., 2017). The polarimetric information therefore can be combined with an arbitrary intensity measure. For example a combination of a constant intensity of one SAR sensor (for study purposes) and an intensity acquired by another SAR sensor or even with a reflectance acquired by panchromatic optical sensors are possible. Because of the incoherent illumination by the sun without fixed polarization direction, polarimetry cannot measured by optical satellite sensors. In summary, the only cross connection between SAR and optics is the total intensity, whereas SAR is able to provide additionally polarimetric information about the illuminated targets.

\subsection{Spectral frame}

The spectral resolution is one key feature of optical sensors. We distinguish panchromatic, multispectral, and hyperspectral sensors. Panchromatic refers to only one image channel with a large bandwidth. Multispectral sensors provide up to 15 bands with medium band width. Hyperspectral images may consist of more than hundred narrow and highly correlated bands. This article focusses on the four channel image which is typical for aerial sensor systems measuring blue, green, red, and infrared reflectance values gathered in vector $\overrightarrow{\mathcal{R}}$. Furthermore, these four bands are delivered in the maximum spatial resolution $(10 \mathrm{~m}$ pixel raster) in the products of Sentinel-2. The goal is the separation of intensity from spectral information which is reached by the traditional Hue-Saturation-Value (HSV) transformation for R-G-B images. We defined an invertible linear transform of four channels which is fully described by the 4-by-4 matrix $\boldsymbol{A}$ (Eq. (1)). Out of the infinite number of possible orthogonal transformations, the elements of $\boldsymbol{A}$ are chosen according to the Kennaugh concept in polarimetry. Following equation with total intensity and intensity differences with equal weighting of positive and negative summands has been defined:

$$
\boldsymbol{A}=\frac{1}{2}\left[\begin{array}{rrrr}
1 & 1 & 1 & 1 \\
-1 & -1 & 1 & 1 \\
-1 & 1 & 1 & -1 \\
-1 & 1 & -1 & 1
\end{array}\right]
$$

Assuming a uniform distribution of the intensity over the four input channels (a grey scale image respectively), the expectation value of each resulting spectral Kennaugh element is zero. By analogy to the polarimetric Kennaugh elements any deviation from zero can be interpreted as spectral information.

From wavelet theory, this transform might be interpreted as Haar wavelet decomposition: the first row contains the low pass, the second row reflects the band pass Haar wavelet of the first scale in central position, the third row contains the same Haar wavelet shifted by one channel, and the fourth row defines the high pass Haar wavelet (Haar, 1910).

Back to matrix calculation, the design matrix $\boldsymbol{A}$ represents an orthogonal matrix which means that it is simply invertible by transposition $\mathbf{E}=\boldsymbol{A} \cdot \boldsymbol{A}^{\mathrm{T}}$. The multiplication with $\boldsymbol{A}$ does not change the length of the input colour vector $\|\mathcal{R}\|=\|\boldsymbol{A} \cdot \mathcal{R}\|$, and the resulting dimensions are orthogonal and thus independent of each other. The linear transform then unfolds to

$$
\overrightarrow{\mathcal{K}}=\left[\begin{array}{l}
\mathcal{K}_{0} \\
\mathcal{K}_{1} \\
\mathcal{K}_{2} \\
\mathcal{K}_{3}
\end{array}\right]=\mathbf{A} \cdot\left[\begin{array}{l}
\mathcal{R}_{\text {blue }} \\
\mathcal{R}_{\text {green }} \\
\mathcal{R}_{\text {red }} \\
\mathcal{R}_{\text {infrared }}
\end{array}\right]=\mathbf{A} \cdot \overrightarrow{\mathcal{R}}
$$

where the elements of $\overrightarrow{\mathcal{K}}$ share the same characteristics as the Kennaugh elements known from polarimetry. Hence, $\mathcal{K}_{0}$ is the total intensity. The remaining elements resemble intensity differences. In this manner, the proposed decomposition are similar to the well-known Tasselled Cap transform with the main difference that the Tasselled Cap reduces the dimensionality and hence does not represent an orthogonal transform (Kauth and Thomas, 1976). All Kennaugh elements can be projected on a closed value range by the division through the total intensity. According to Schmitt et al. (2015) the normalized elements can be defined as follows

$$
\begin{aligned}
& \left.k_{0}=\left(\mathcal{K}_{0}-1\right) /\left(\mathcal{K}_{0}+1\right) \in\right]-1,+1[ \\
& \left.k_{\mathrm{i}}=\mathcal{K}_{\mathrm{i}} / \mathcal{K}_{0} \text { for } \mathrm{i}=1,2,3 \in\right]-1,+1[
\end{aligned}
$$

In consequence, these multi-spectral elements can be treated as Kennaugh elements known from polarimetry. The inverse transform is always possible applying $\overrightarrow{\mathcal{R}}=\mathbf{A}^{\mathrm{T}} \cdot \overrightarrow{\mathcal{K}}$. The presented orthogonal transform allows the separation of intensity from multispectral information. As the monofrequency SAR sensors in general are not able to provide multispectral information, the only cross connection between SAR and optical data again is given by the total intensity.

\section{SAR-SHARPENING}

Thanks to the chosen geometric and radiometric frames, the fusion requirements are already fulfilled by the pre-processing steps. The delivered SAR data processed in the Multi-SAR framework (Bertram et al., 2016), the optical data sets provided by the Sentinel-2 mission, and the aerial image mosaic (LDBV 2018) can directly be used. Minor deviations resulting from an outdated or coarse digital elevation model might potentially occur but are not addressed in this article. The question to be answered in the following sections is how to optimally fuse intensity measurements and how to replace intensity channels without influencing polarimetry and spectral properties in a multi-sensor data set.

\subsection{Intensity Averaging}

Intensity by definition represents a conservative potential field. For instance, there is no negative intensity and the mean intensity of an area - defined as the arithmetic mean of the available local intensity measures - is always greater than zero. Hence, an 
additive combination of intensity measures is prescribed. The polarimetric and spectral Kennaugh decomposition in this sense is nothing else than a linear combination of intensities. The Kennaugh elements, be it $K_{i}$ or $\mathcal{K}_{i}$, can be treated in the same way. In order to consider the potentially varying spatial resolution of the input data, the number of looks $l_{j}$ per pixel is introduced as weight. Assuming $n$ intensity images of the same area, the total number of looks is given by $l=\sum_{j=1}^{n} l_{j}$. From statistics, this can be interpreted as the mean over $l$ independent measurements available for the target pixel area. The individual number of looks $l_{j}$ can be seen as quotient of the target pixel area $a_{t}$ in the fused image by the measured pixel area $a_{m, j}$ and provides an adequate sampling rate:

$$
\mathrm{l}_{\mathrm{j}}=\mathrm{a}_{\mathrm{t}} / \mathrm{a}_{\mathrm{m}, \mathrm{j}}
$$

The intensity fusion hence unfolds to the weighted arithmetic mean of the input intensities $K_{i, j}$ in linear scale including the look numbers as weights:

$$
\mathrm{sK}_{\mathrm{i}}=\frac{1}{\mathrm{l}} \sum_{\mathrm{j}=1}^{\mathrm{n}} \mathrm{l}_{\mathrm{j}} \cdot \mathrm{K}_{\mathrm{i}, \mathrm{j}}
$$

The fused intensity $s K_{0}$ is given in linear scale again, i.e. $s K_{0} \in[0, \infty[$. This is also the typical data range of variance measures. From radar theory, any intensity resembles a squared deviation. The mean intensity over $l$ measurements hence defines the mean squared deviation, namely the variance.

As the use of normalized intensities is preferable with view to memory demand (Schmitt et al., 2015), the following equation can be derived from Eq. 3 for the fused normalized intensity which is independent from polarimetric or spectral information:

$$
s k_{0}=\frac{s K_{0}-1}{s K_{0}+1}=\frac{\sum_{j=1}^{n} \frac{l_{j}}{1-k_{0, j}} k_{0, j}}{\sum_{j=1}^{n} \frac{l_{j}}{1-k_{0, j}}}
$$

In that way, the workaround over linear intensities can be avoided. The normalized fused intensity shows a closed value range $\left.s k_{0} \in\right]-1,+1[$. With respect to statistics, the fused intensity $s k_{0}$ equals the normalized deviation from a normal distribution with its expected variance in one.

Regarding the definition of normalized polarimetric and spectral Kennaugh elements respectively in Eq. 4, the calculation of the fused elements consequently unfolds to

$$
s k_{i}=\frac{s K_{i}}{s K_{0}}=\frac{\sum_{j=1}^{n} l_{j} \cdot K_{i, j}}{\sum_{j=1}^{n} l_{j} \cdot K_{0, j}}=\frac{\sum_{j=1}^{n} \frac{l_{j}+l_{j} \cdot k_{0, j}}{1-k_{0, j}} \cdot k_{i, j}}{\sum_{j=1}^{n} \frac{l_{j}+l_{j} \cdot k_{0, j}}{1-k_{0, j}}} \quad i>0
$$

In summary, fused intensity, polarimetric, and spectral information can be expressed in Kennaugh elements in linear and in TANH scale. The additive fusion as weighted arithmetic (see Eq. 6) yields maximum stability for statistical reasons as long as the images to be fused share exactly the same polarimetric or spectral dimensions.

\subsection{Intensity Substitution}

The idea behind the Kennaugh decomposition is the separation of intensity from polarimetric and/or spectral information in order to remove illumination effects like insufficient topographic calibration in SAR images or varying solar irradiance in optical images. The image then decomposes to

$$
\vec{I}_{S A R}=\left[\begin{array}{c}
K_{0} \\
K_{1} \\
\vdots \\
K_{m}
\end{array}\right]=K_{0} \cdot\left[\begin{array}{c}
1 \\
k_{1} \\
\vdots \\
k_{m}
\end{array}\right] \text { or } \vec{I}_{O P T}=\left[\begin{array}{c}
\mathcal{K}_{0} \\
\mathcal{K}_{1} \\
\vdots \\
\mathcal{K}_{q}
\end{array}\right]=\mathcal{K}_{0} \cdot\left[\begin{array}{c}
1 \\
k_{1} \\
\vdots \\
k_{q}
\end{array}\right]
$$

Both the scalar intensity and the Kennaugh vector can be substituted. For instance, the polarimetry acquired by a SAR sensor can be spread by the intensity measured by an optical sensor in order to retrieve smoother results. The spectral Kennaugh elements of an optical image can be stretched by the intensity acquired by a SAR sensor in order introduce image texture vice versa. The intensity is the only overlapping dimension as stated before. Hence, both intensity measures can potentially be fused according to Eq. 6 whereas the vectors of polarimetric and spectral elements (see Eq. 9) are just concatenated:

$$
I_{S A R \& O P T}=s K_{0} \cdot\left[\begin{array}{lllllll}
1 & k_{1} & \cdots & k_{m} & k_{1} & \cdots & k_{q}
\end{array}\right]^{T}
$$

As only the intensity measure is fused, this approach is reasonable if images with no overlap in the polarimetric or spectral domain are available. The typical application is the fusion of a multipolarized SAR image with a multi-spectral optical image.

\subsection{Intensity Fusion}

The most general and most complicated case is the fusion of several partially overlapping dimensions. In contrast to the preceding sections, both requirements have to be fulfilled at the same time: the stable additive combination from (Sec. 4.1) and the isolated consideration of intensity and polarimetric/spectral information from (Sec. 4.2). According to Eqs. 2 and 6, the fusion of linear Kennaugh elements can be expressed in matrix notation:

$$
s \overrightarrow{\mathcal{K}}=\frac{1}{l} \sum_{j=1}^{n} l_{j} \cdot \overrightarrow{\mathcal{K}}_{j}=\frac{1}{l} \sum_{j=1}^{n} l_{j} \cdot \boldsymbol{A} \cdot \overrightarrow{\mathcal{R}}_{j}=\frac{A}{l} \sum_{j=1}^{n} l_{j} \cdot \overrightarrow{\mathcal{R}}_{j}
$$

Obviously, it is completely irrelevant whether a collection of Kennaugh vectors $\overrightarrow{\mathcal{K}}_{\mathrm{j}}$ or a collection of reflectance vectors $\overrightarrow{\mathcal{R}}_{\mathrm{j}}$ is fused. Assuming that not all positions of $\overrightarrow{\mathcal{R}}$ or $\overrightarrow{\mathcal{K}}$ are filled, the entity of measurements and the total number of looks l needed for normalization purposes is no longer uniform. That is why a look vector $\vec{l}_{j}$ with entries $l_{i, j}$ is introduced that attaches an individual look number to each element $\mathcal{K}_{i, j}$ of $\overrightarrow{\mathcal{K}}_{\mathrm{j}}$. The normalization leads to an elements-wise division by the corresponding look number.

$$
s \mathcal{K}_{i}=\frac{\sum_{j=1}^{n} l_{i, j} \mathcal{K}_{i, j}}{\sum_{j=1}^{n} l_{i, j}}
$$

The same problem occurs with the normalized Kennaugh elements: the total intensity $s \mathcal{K}_{0}$ as weighted sum over all measurements $j$ is not the adequate calibration factor for all entries $s \mathcal{K}_{i}$ because $s \mathcal{K}_{i}$ possibly composes of only a subset of all measurements. This is taken into account by the individual look number $l_{i, j}$ and a specific total intensity for each polarimetric/spectral element:

$$
s k_{i}=\frac{\sum_{j=1}^{n} K_{0, j} \cdot l_{i, j} \cdot k_{i, j}}{\sum_{j=1}^{n} K_{0, j} \cdot l_{i, j}} \quad i>0
$$

The total intensity $s k_{0}$ which is the essential dimension of each measurement is calculated by applying the look numbers $l_{0, j}$ which are identical to $l_{j}$ known from Eq. 11. The normalization by the reference intensity of one finally leads to

$$
s k_{0}=\frac{\sum_{j=1}^{n} l_{0, j} \cdot K_{0, j}-\sum_{j=1}^{n} l_{0, j}}{\sum_{j=1}^{n} l_{0, j} \cdot K_{0, j}+\sum_{j=1}^{n} l_{0, j}}
$$

In summary, three cases of data fusion have been addressed: the averaging of redundant measurements (as mathematical basis for the whole data fusion approach), the substitution of independent measurements (scenarios 3 and 4), and the fusion of partially redundant measurements (scenarios 1 and 2). Those cases will be subject to the following application and quality assessment. 


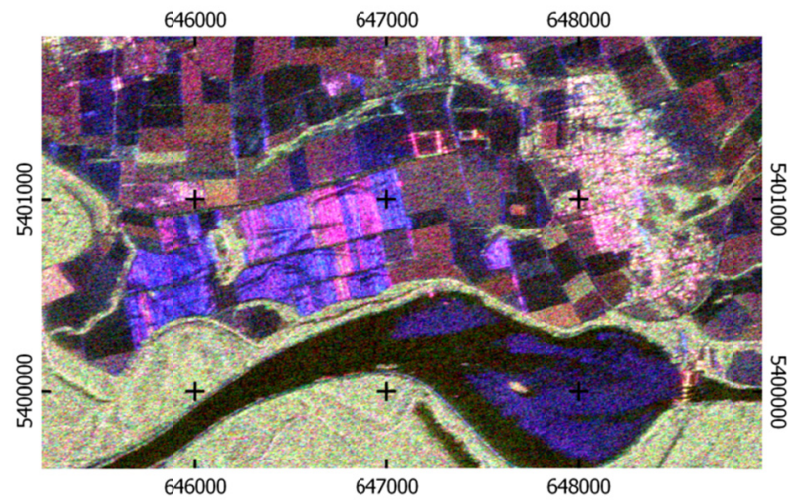

ALOS-PALSAR-2 Pauli (12.05.2017) (CJAXA 2017

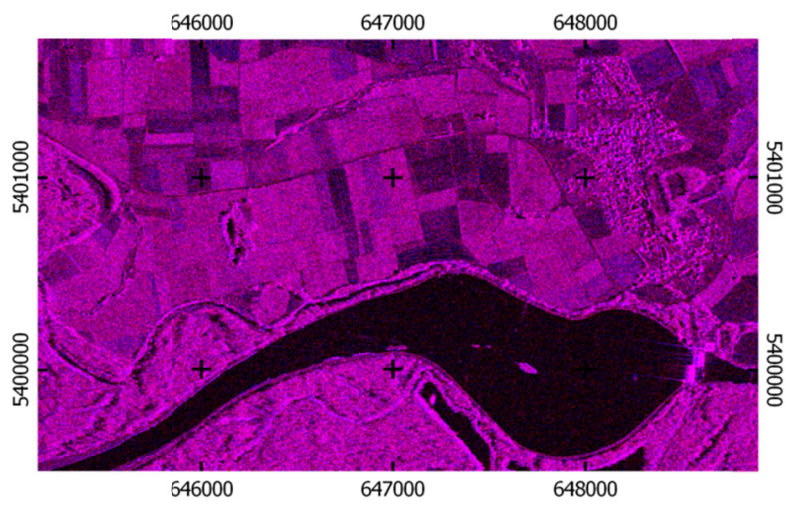

TerraSAR-X HH VV (20.04.2017) @DLR 2017

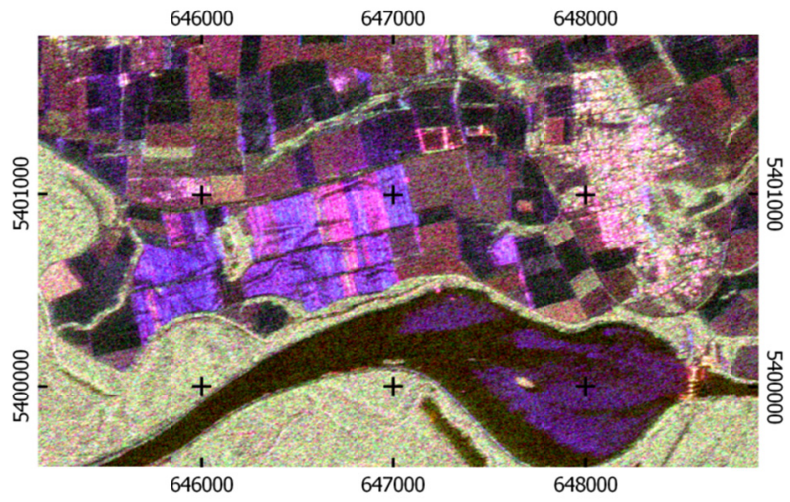

Fused image in Pauli decomposition

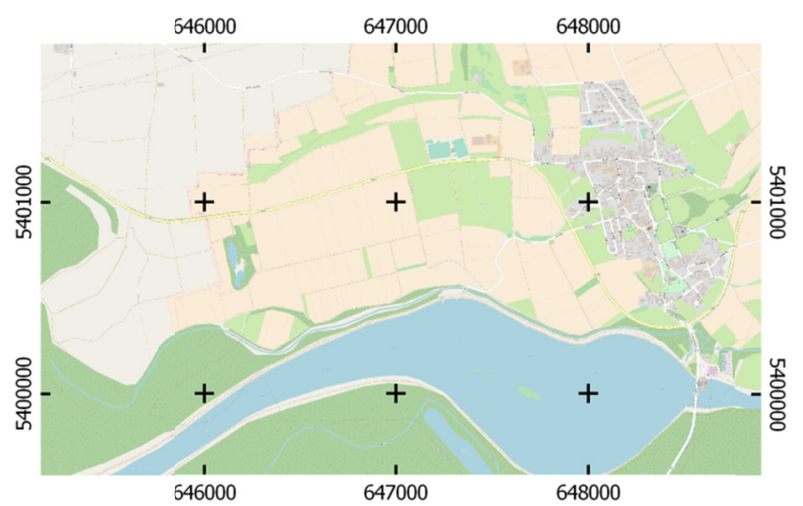

OSM Reference (c) OpenStreetMap contributors

Figure 1. The fusion of dual-co-pol and quad-pol images.

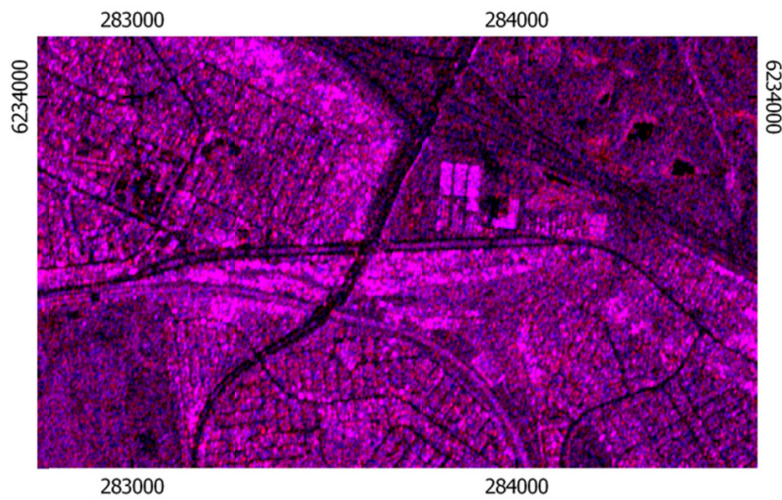

TerraSAR-X HH VV (22.11.2014) CDLR 2014

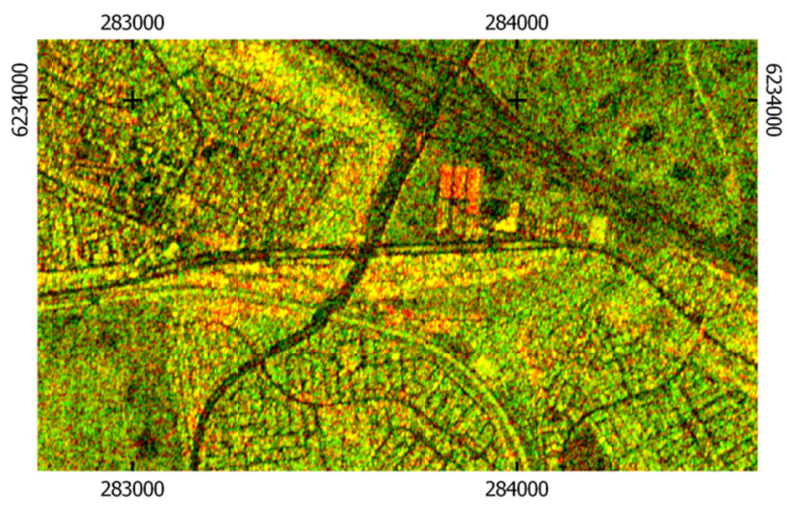

TerraSAR-X VV VH (03.12.2014) CDLR 2014

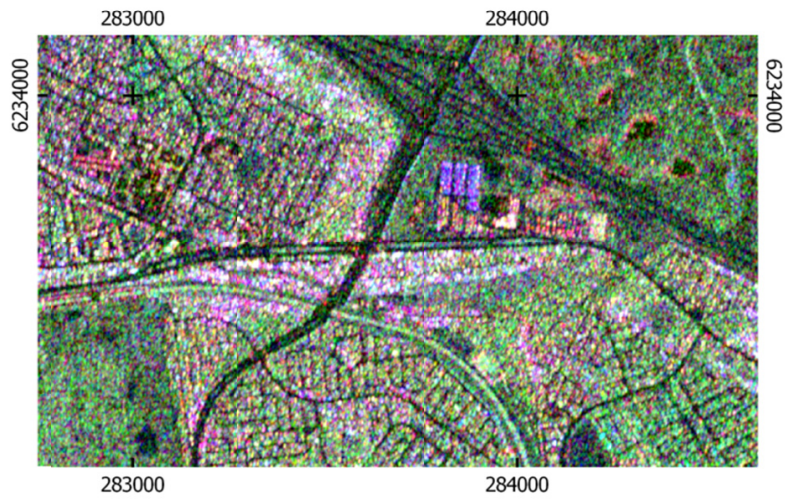

Fused Quadpol image in Pauli decomposition

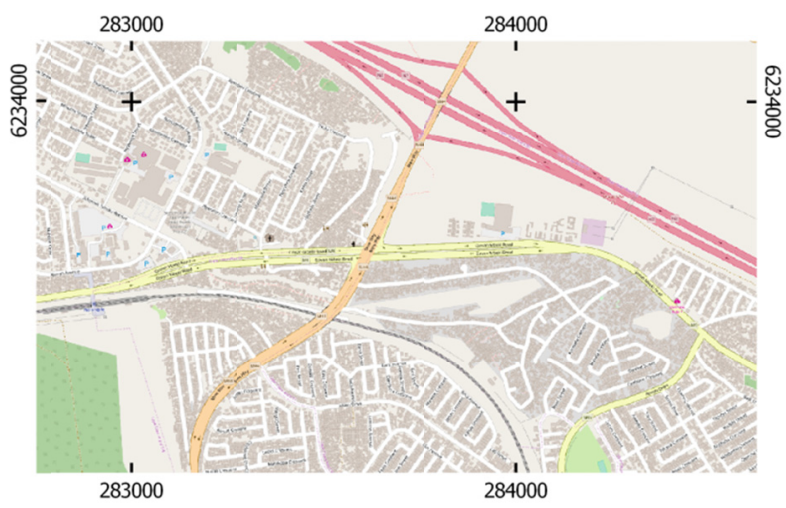

OSM Reference (c) OpenStreetMap contributors

Figure 2. The fusion of dual-pol images to one quad-pol image. 

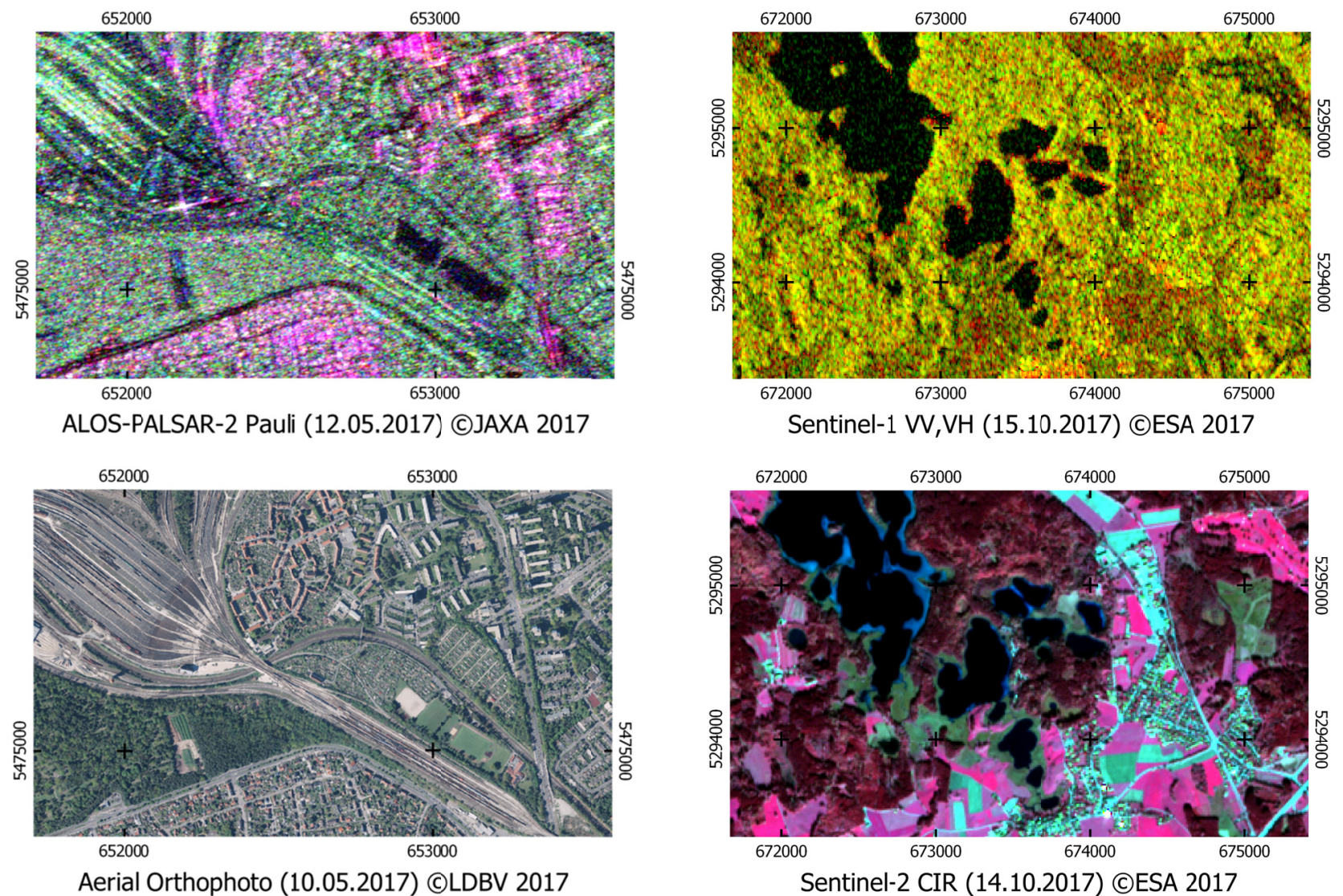

Aerial Orthophoto (10.05.2017) (CLDBV 2017
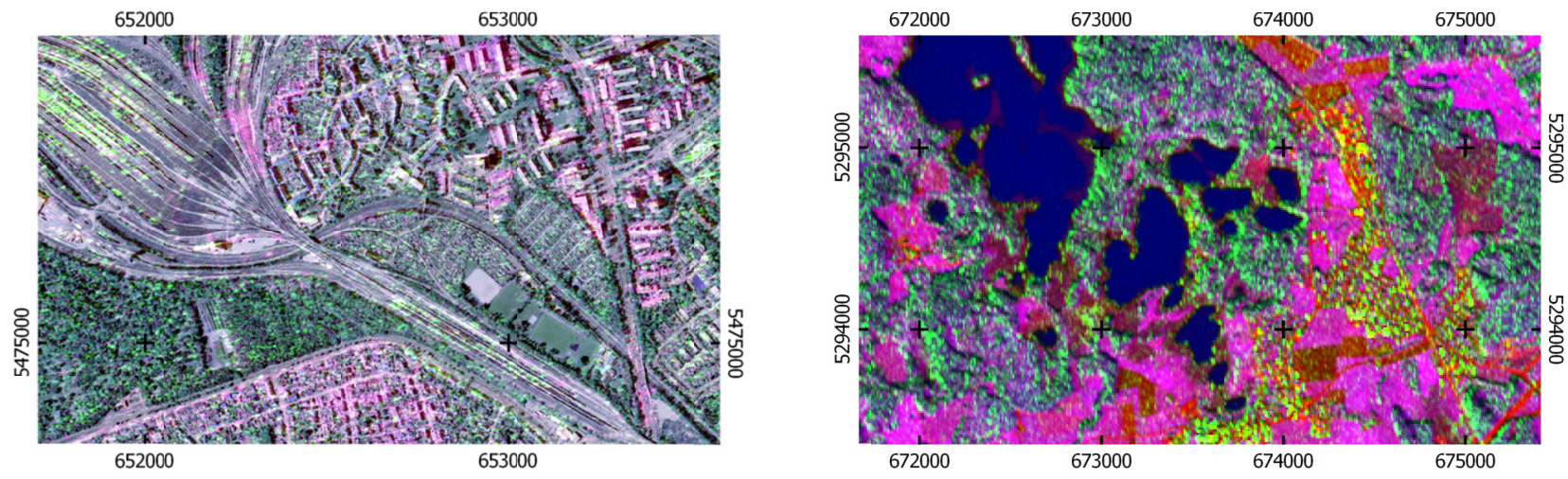

Fused SAR\&Optics Pauli/RGB image

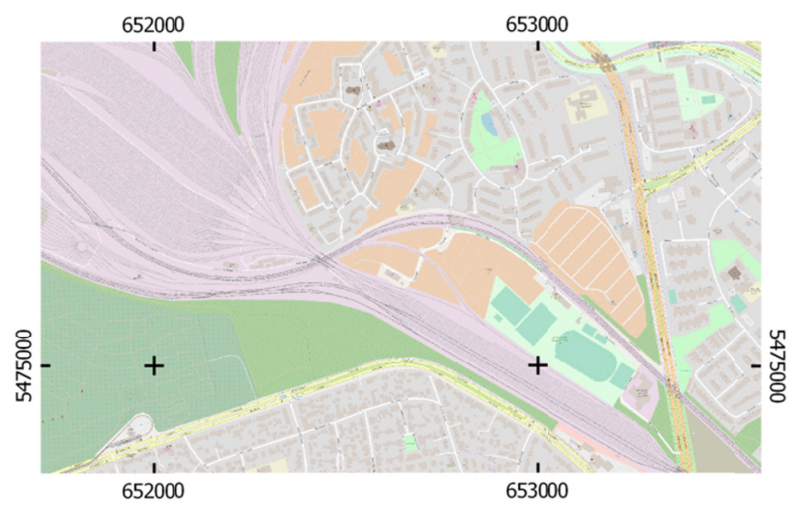

OSM Reference (c) OpenStreetMap contributors

Figure 3. The fusion of quad-pol SAR with Aerial Orthophotos.

Sentinel-1\&-2 intensity and spectral content CCESA 2017

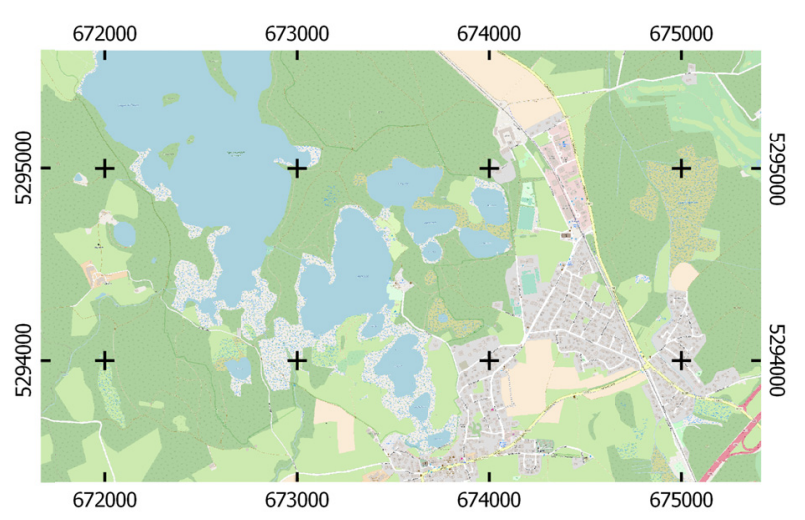

OSM Reference (c) OpenStreetMap contributors

Figure 4. The fusion of Sentinel-1 and Sentinel-2. 


\section{RESULTS}

This section illustrates the results of the data fusion approach: Scenario 1 - A quad-pol image acquisition of ALOS-PALSAR-2 is fused with a dual-co-pol spotlight image of TerraSAR-X in order to slightly enhance the spatial resolution and to stabilize the co-polarized information according to Sec. 4.3, see Fig. 1.

Scenario 2 - Two dual-pol stripmap acquisitions of TerraSAR-X, namely one dual-co-pol $\mathrm{HH} / \mathrm{VV}$ and one dual-cross-pol VV/VH measurement, are fused in order to generate a synthetic, but adequate quad-pol image according to Sec. 4.3, see Fig. 2.

Scenario 3 - The intensity of a quad-pol image acquired by ALOS-PALSAR-2 is replaced by the total intensity of the channels measured by an airborne camera in order to enhance the spatial resolution according to Sec. 4.2, see Fig. 3.

Scenario 4 - The images of the Sentinel-1 (Interferometric Wide Swath, VV/VH) and Sentinel-2 (Blue-Green-Red-Infrared) missions are fused in order to introduce SAR texture into the multispectral image according to Sec. 4.2, see Fig. 4.

Figs 1-4 depict the input images, the fused data set, and a physical map of the respective test site. The coordinates refer to UTM Zone 32N and UTM Zone 34S respectively for Fig. 2.

\section{VALIDATION}

The validation of image fusion algorithms is always a difficult task for lack of adequate and comprehensive ground truth data. Consequently, inter-comparison is the only feasible way. As input images inherently differ in terms of sensor, wavelength, illumination, and image generation, just to mention a few aspects, measures that match both the input and the fused images are required. We decided in favour of two isolated considerations: first, spatial resolution and second, polarimetric resolution.

Spatial resolution is described by the local gradient: the higher the gradient, the higher the resolution as long as the mean values are not contaminated by noise. The noise contamination comes along with a random change of the local value. Therefore, the local intensity is plotted against the local gradient according to Schmitt (2016). The left-hand side of Figs. 5-8 illustrates the distribution of the two input images in red and green and the resulting distribution of the fused images in blue. The polarimetric resolution, generally called "polarimetric information content", is determined in the entropy-alpha plane. Entropy shows the diversity of the local scattering, whereas the alpha angle indicates the location of the mean backscattering in the polarimetric domain and thus the scattering mechanism (Cloude and Pottier, 1996). Depending on the input polarizations the scatter plot in the entropy-alpha plane shows varying characteristics. In general, the scatter data range varies from narrow to broad band with increasing polarimetric information (Cloude, 2007). The distribution is again plotted in three colors: red and green for the input images, and blue for the fused image.

Mixed colors display the joint occurrence in two images, whereas pink stands for an overlay of the fused image with the first input image and turquoise for the accordance between the fused image and the second input image. White demonstrates that all three images share a high occurrence in the local feature plane. Pure red or green color means that features of the input images are dismissed in the fused image. Pure blue marks new information. The validation of scenario 1 in Fig. 5 shows that the distribution of the mean and the gradient is quite different in the two input images. Nevertheless, the fused image is a good trade-off between both input intensities: the overlay of TerraSAR-X and ALOSPALSAR-2 is completely covered by the fused image. Additionally, both the pink and turquoise areas can be identified, where the characteristics of one input image are captured. With respect to the polarimetric resolution on the right side of Fig. 5, the input images fill a small part of the feature plane, whereas the fused image covers nearly the whole of the possible data range. The validation of scenario 2 in Fig. 6 suggests that the input images are quite similar in terms of mean and gradient which is reasonable because both images are acquired by TerraSAR-X in the same acquisition mode. The fused image necessarily shares the same characteristics. Regarding the polarimetric properties on the right-hand side of Fig. 6, the polarimetric information contained in the dual-co-pol and dual-cross-pol images is quite different. Nevertheless, the fused image fills the whole data range, hence it optimally integrates both partial-polarimetric information.

The validation of scenario 3 in Fig. 7 shows the intensity fusion whilst preserving the polarimetric properties. Both requirements are perfectly met by the fused image. The distribution of the mean and the gradient matches the distribution of the optical input image. The polarimetric information is completely identical to the quad-pol input image. Hence, the proposed image fusion guarantees the separation of intensity and polarimetry.

A similar behaviour can be observed in the validation of scenario 4 in Fig. 8. The polarimetric distribution of the fused image follows the distribution of the SAR input image independent of the spectral information content introduced by the
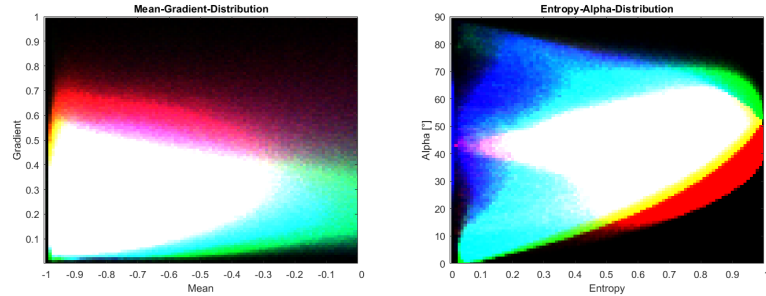

Figure 5. 2D-distribution of TerraSAR-X dual-co-pol (red), ALOS-PALSAR quad-pol (green), and the fused image (blue).
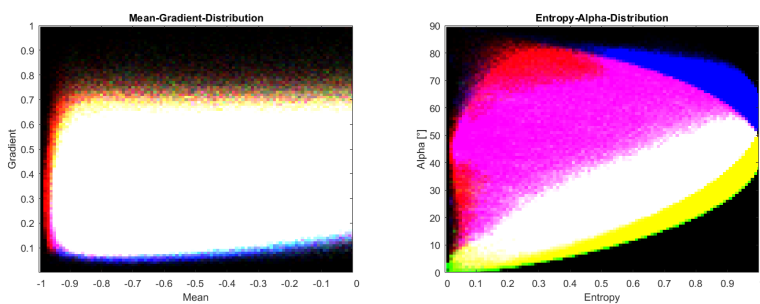

Figure 6. 2D-distribution of TerraSAR-X dual-co-pol (red), dual-cross-pol (green), and the combined image (blue).
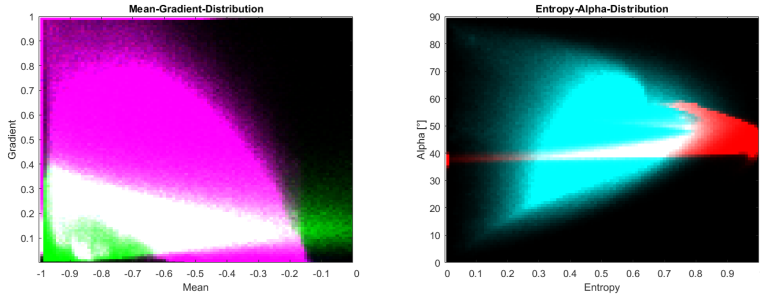

Figure 7. 2D-distribution of an aerial orthophoto (red), ALOSPALSAR quad-pol (green), and the fused image (blue).
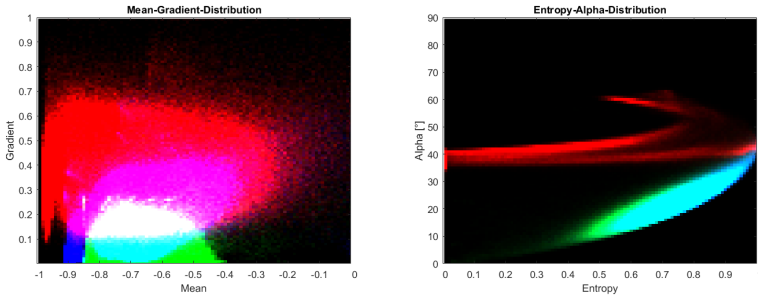

Figure 8. 2D-distribution of Sentinel-2 R-G-B-IR (red), Sentinel-1 VV/VH (green), and the fused image (blue). 
optical input image. The validation of the mean and the gradient indicates that the image characteristics of the input acquisitions are very different, which was expected (e.g. Fig. 7). In contrast to scenario 3 , the fused image does not follow the optical intensity exclusively because of the similar spatial resolution - and thus, almost equal look numbers - with Sentinel-1 and Sentinel-2 images. Therefore, the fused intensity reflects an improved mean of both inputs. In summary, the proposed image fusion algorithm fulfils all requirements in each of the four scenarios which cover varying multi-sensor input data as well as varying test sites.

\section{CONCLUSION}

This article introduces a versatile approach to SAR-Sharpening in analogy to PAN-Sharpening known from optical data. It is based on the Kennaugh framework known from SAR pre-processing. The geometric frame is given by geocoded images in earth-fixed coordinates. The radiometric frame refers to the horizontal projection plane which requires $\sigma_{0}$. The polarimetric frame is given by the normalized Kennaugh elements decomposing multipolarized measurements into a total intensity referred to one and several normalized intensity differences. With respect to optical images, multi-spectral Kennaugh elements are defined for the first time. They share the same properties with polarimetric Kennaugh elements and thus guarantee the easy fusion of SAR and optical data sets. The fusion of partial measurements takes into account the local number of data points and the backscatter intensity, which refers to the reliability of the derived polarimetric or spectral information. The normalization step always has to comply with the total intensity of the corresponding Kennaugh element. The general definition simplifies in case of completely overlapping polarimetric and/or spectral domain or in the case of a pure intensity fusion. The validation considers the mean and the gradient of the fused intensity as well as the polarimetric information content depicted in the entropy-alpha plane. The four scenarios prove that the separation of intensity and polarimetric/spectral information is achieved on one hand, and the fused images optimally integrate the information provided by both input data sets on the other hand. This approach completes the Kennaugh framework previously introduced for the preprocessing of multi-sensor SAR data and the robust change detection. It opens the door to the Kennaugh processing of optical data sets and thus, brings SAR and Optical remote sensing another small step closer.

\section{ACKNOWLEDGEMENTS}

The authors acknowledge the use of TerraSAR-X data (CDLR 2014 \& 2017), Sentinel-1 and Sentinel-2 data (CESA 2017), ALOS-PALSAR-2 data (CJAXA 2017), and aerial orthophotos (CGeobasisdaten: Bayerische Vermessungsverwaltung).

\section{REFERENCES}

Abdikan, S., Balik Sanli, F., Bektas Balcik, F., and Goksel, C., 2008. Fusion of SAR images (PALSAR and RADARSAT-1) with multispectral spot image: a comparative analysis of resulting images. In: Int. Arch. Photogramm. Remote Sens. Spatial Inf. Sci., 37, 1197-1202.

Amarsaikhan, D., Blotevogel, H.H., van Genderen, J.L., Ganzorig, M., Gantuya, R., and Nergui, B., 2010. Fusing high-resolution SAR and optical imagery for improved urban land cover study and classification. Int. J. of Image and Data Fusion, 1:1, 83-97.

Bertram, A., Wendleder, A., Schmitt, A., and Huber, M., 2016. Long-term Monitoring of water dynamics in the Sahel region using the Multi-SARSystem. In: Int. Arch. Photogramm. Remote Sens. Spatial Inf. Sci., XLIB8, 313-320, doi.org/10.5194/isprs-archives-XLI-B8-313-2016.

Brown, L.G., 1992. A Survey of Image Registration Techniques. ACM Computing Surveys, 24 (4), 325-376.

Chavez, P. S., Sides, S.C., Anderson, J. A., 1991. Comparison of three different methods to merge multiresolution and multispectral data: TM \& SPOT pan. Photogram. Engineering and Remote Sensing, 57, 295-303.
Cliche, G., Bonn, F., Teillet, P., 1985. Integration of the SPOT Pan channel into its multispectral mode for image sharpness enhancement. Photogrammetric Engineering and Remote Sensing, 51, 311-316.

Cloude, R.C., 2007. The dual polarisation entropy/alpha decomposition: a PALSAR case study. In: Proc. PolInSAR 2007, Frascati, Italy. http://earth .esa.int/workshops/polinsar2007/papers/75_cloude.pdf (28 March 2018).

Cloude, R.C. and Pottier, E., 1996. A Review of Target Decomposition Theorems in Radar Polarimetry. IEEE Trans. of Geosc. and Remote Sensing, 34 (2), 498-518.

ESA, 2018. Sentinel-2 products: Level-2A Algorithm Overview https://sentinel.esa.int/web/sentinel/technical-guides/sentinel-2-msi/level2a/algorithm (28 March 2018).

Farina, A., Costantini, M., Zirilli, F., 1996. Fusion of radar images: techniques and applications. Military Technology (Miltech), 5, 34-40.

Haar, A., 1910. Zur Theorie der orthogonalen Funktionensysteme Mathematische Annalen 69, 331-371. https://link.springer.com/ article/10.1007\%2FBF01456927 (28 March 2018).

Kauth, R.J. and Thomas, G.S., 1976. The Tasseled Cap - A Graphic Description of the Spectral-Temporal Development of Agricultural Crops as Seen by LANDSAT. In: LARS Symposia. 159. http://docs.lib.purdue.edu/lars symp/159 (28 March 2018).

LDBV, 2018. Luftbilder - Hochauflösende Senkrechtaufnahmen der Erdoberfläche. https://www.ldbv.bayern.de/produkte/luftbild/luftbilder.html.

Li, Y., Hong, W., Pottier, E., 2015. Topography retrieval from single-pass POLSAR data based on the polarization-dependent intensity ratio. IEEE Transactions on Geoscience and Remote Sensing, 53 (6), 3160-3177. doi.org/10.1109/TGRS.2014.23694817.

Moreira, A., Prats-Iraola, P., Younis, M., Krieger, G., Hajnsek, I., and Papathanassiou, K.P., 2013. A Tutorial on Synthetic Aperture Radar. IEEE Geoscience and Remote Sensing Magazine, 1 (1), 6-43. doi.org/10.1109/MGRS.2013.2248301

Moser, L., Schmitt, A., Wendleder, A., 2016. Automated wetland delineation from multi-frequency and multi-polarized SAR images in high temporal and spatial resolution. In: Int. Ann. Photogramm. Remote Sens. Spatial Inf. Sci., III (8), 57-64.

Moser, L., Schmitt, A., Wendleder, A., and Roth, A., 2015. Monitoring of the Lac Bam Wetland Using Dual-Polarized X-Band SAR Data. MDPI Remote Sensing, 8 (302), 1-31.

Peter, H., Jäggi, A., Fernández, J., Escobar, D., Ayuga, F., Arnold, D., Wermuth, M., Hackel, S., Otten, M., Simons, W., Visser, P., Hugentobler, U., and Féménias, P., 2017. Sentinel-1A - First precise orbit determination results. Advances in Space Research, 60, 879-892.

Pohl, C. and van Genderen, J.L., 1998. Multisensor image fusion in remote sensing: concepts, methods and applications. Int. J. Remote Sensing, 19 (5), pp. 823-854.

Schmitt, A., 2016. Multiscale and Multidirectional Multilooking for SAR Image Enhancement. IEEE Transactions on Geoscience and Remote Sensing, 54 (9), $\quad 5117-5134$. doi.org/10.1109/TGRS.2016.2555624

Schmitt, A., Wendleder, A., Hinz, S., 2015. The Kennaugh element framework for multi-scale, multi-polarized, multi-temporal and multifrequency SAR image preparation. ISPRS J. of Photogrammetry and Remote Sensing, 102, 122-139.

Schmitt, A. and Brisco, B., 2013. Wetland Monitoring Using the Curvelet-Based Change Detection Method on Polarimetric SAR Imagery. MDPI Water, 5, 1036-1051. ISSN 2073-4441 doi.org/10.3390/w5031036.

Schubert, A., Small, D., Miranda, N., Geudtner, D., and Meier, E., 2015. Sentinel-1A Product Geolocation Accuracy: Commissioning Phase Results. In: MDPI Remote Sensing, 7, 9431-9449. doi.org/10.3390/rs70709431.

Simone, G., Morabite, F., Farina, A., 2001. Multifrequency and multiresolution fusion of SAR images for remote sensing applications In: Proceedings of $3^{\text {rd }}$ IEEE International Conference on Fusion, FUSION 2000, Paris, France, July 10-13, 2000, pp. WeD3.10-17.

Small, D., 2011. Flattening Gamma: Radiometric Terrain Correction for SAR Imagery. IEEE Transactions of Geoscience and Remote Sensing, 49 (8), 3081-3093. doi.org/10.1109/TGRS.2011.2120616.

Ullmann, T., Banks, S.N., Schmitt, A., and Jagdhuber, T., 2017. Scattering Characteristics of $\mathrm{X}-, \mathrm{C}-$ and L-Band PolSAR Data Examined for the Tundra Environment of the Tuktoyaktuk Peninsula, Canada. MDPI Applied Sciences, 7 (6), 595.

Vajsova, B. and Åstrand, P.J., 2015. New sensors benchmark report on Sentinel-2A. Joint Research Center Technical Reports. http://publications.jrc.ec.europa.eu/repository/bitstream/JRC99517/lbna-27674-en-n\%20.pdf (28 March 2018).

Weissgerber, F., 2016. Resolution enhancement of polarimetric images using a high resolution mono-channel image. In: $11^{\text {th }}$ EUSAR Conference, Hamburg, Germany. 